Article

\title{
Self-Assembled Fluorinated Organogelators for Surface Modification
}

\section{Anilkumar Raghavanpillai * and Vincent A. Franco}

DuPont Central Research \& Development, Experimental Station, Wilmington, DE 19880, USA; E-Mail: vincent.a.franco@usa.dupont.com

* Author to whom correspondence should be addressed;

E-Mail: anilkumar.raghavanpillai@usa.dupont.com; Tel.: +1-302-695-6846;

Fax: +1-302-695-2112.

Received: 16 January 2012; in revised form: 15 February 2012 / Accepted: 21 February 2012 / Published: 1 March 2012

\begin{abstract}
A new class of alkyl- and perfluoroalkyl-containing urea and amide derivatives was synthesized from amino acid derivatives. Most of these compounds showed excellent gelation behavior in organic solvents at low concentrations. A few organogelators selected from the initial screening were used for surface modification of fibrous substrates to create hydrophobic and oleophobic composites. The hydrophobic and oleophobic behaviors of these composites were ascribed to a combination of increased surface roughness and the alkyl/fluorinated functionalities present in the gelator backbone.
\end{abstract}

Keywords: organogelator; gelation; perfluoroalkyl; urea; amide; amino acid; xerogel; nonwoven; hydrophobic; oleophobic; contact angle; surface modification

\section{Introduction}

Low molecular weight organogelators (LMWOGs) are gaining increased attention due to their potential applications in a variety of industrial sectors related to materials, cosmetics, health care, food and oil technology [1-9]. The design and elucidation of structure-property relationships of gelators is also a valuable area of research, in particular, establishing the relationship between the chemical structure of a gelator and its gelation properties in a given solvent [10-18]. Thermally reversible physical gels are generally formed by self-assembly of the gelator molecules in solvent, leading to the 
formation of a fibrous supramolecular network with the encapsulation of solvent. This complex process of self-assembly into supramolecular structures varies based on the functionalities present in the organogelator and external factors, including solvent, temperature, and $\mathrm{pH}$. Various non-covalent interactions, such as hydrogen bonding, $\pi$ - $\pi$ stacking, hydrophobic interactions, and metal coordination, influence the formation and stabilization of the organogelator self-assemblies [1,2,10-14,19-21]. However, the non-covalent forces influencing gelation are relatively weak compared to covalent bonds, and a combination of many of these interactions is required to form a strong intermolecular network. Each molecule of an organogelator can establish several types of physical interactions with a neighboring molecule. For example, intermolecular hydrogen bonding usually facilitates the growth of linear, elongated aggregates with solvent entrapment. Evaporation of solvent from a gel results in the formation of a dried gel (xerogel) with a fibrous or web-like morphology [21]. It is possible to alter the surface morphology of the substrate, and thus surface properties, if the gelation and subsequent evaporation of solvent are performed on a substrate. Hence, gel impregnation methodology could provide the low surface energies desired for fabricating superhydrophobic surfaces [19,21-24]. Surfaces on which the contact angle of water exceeds $150^{\circ}$, and shows low contact angle hysteresis, are described as superhydrophobic or ultrahydrophobic [25-27]. These surfaces repel water like a lotus leaf and are also considered 'self-cleaning', since any surface contamination is removed by water as the droplets roll across the surface [28-30]. The self-cleaning ability of these surfaces is largely due to the increased surface area created by a two-tier micro and nano roughness morphology. It is possible to incorporate nano- or microscale morphologies on any substrate via organogelator impregnation, which could be further controlled by suitable gelling solvents, as well as concentration and temperature effects. In addition, incorporation of low surface energy functionalities like fluorinated groups in the gelator framework could further lower the surface energy and provide oleophobic properties to the modified surface [31].

Urea and amide derivatives are exceptionally well-suited candidates for the design of low molecular weight gelators owing to their rigidity, strength and ability to form highly directional hydrogen bonds [32]. We recently demonstrated this concept by fabricating low surface energy surfaces via the surface modification of nonwoven substrates using partially fluorinated urea-amide gelators [33]. The general structure and examples of these urea-amide gelators reported in that study are shown Figure 1.

Figure 1. General structure and examples of partially fluorinated urea-amide gelators described in [31].

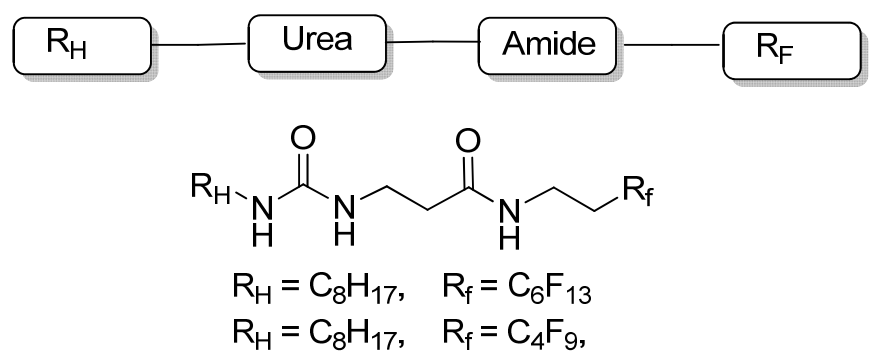

The compounds depicted in Figure 1 were prepared via amidation of the $N$-Boc protected amino acid with fluorinated amines, followed by deprotection and reaction with an alkyl isocynate. They showed excellent gelation behavior in organic solvents. Gelation in the presence of a nonwoven 
substrate led to a gel-impregnated surface, which upon drying produced a composite with porous microstructure morphology. The resulting composite also showed high water and hexadecane contact angles, indicative of excellent hydrophobic and oleophobic properties. Herein, we discuss the design and synthesis of two novel classes of partially fluorinated amide and urea-amide derivatives, where the positions of the hydrocarbon and fluorocarbon tail are reversed in the molecular structure in comparison to the gelator described in Figure 1. General structures of these partially fluorinated amide and urea-amide derivatives are shown in Figure 2. This report also describes the gelation behavior as a function of structural/functional variations of these derivatives and surface modification of nonwoven substrates using them.

Figure 2. General structure of partially fluorinated amide and urea-amide derivatives described in this study.

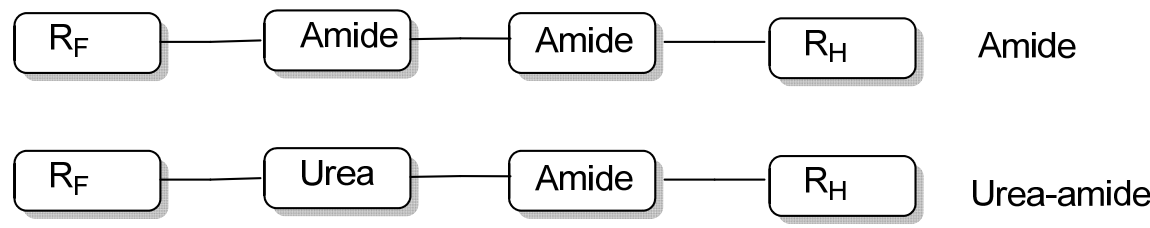

\section{Experimental Section}

\subsection{General}

All solvents and reagents, unless otherwise indicated, were purchased from commercial sources and used directly as supplied. Tyvek ${ }^{\circledR}$ polyethylene nonwoven fabric was obtained from E. I. du Pont de Nemours and Company, Wilmington, DE, and Kolon ${ }^{\circledR}$ gsm70 spun-bonded polyester fabric was obtained from Korea Vilene, Inc. ${ }^{1} \mathrm{H}$ and ${ }^{19} \mathrm{~F}$ NMR spectra were recorded on a Bruker DRX 400 or 500 spectrometer. Chemical shifts are reported in ppm relative to an internal reference $\left(\mathrm{CDCl}_{3}, \mathrm{CFCl}_{3}\right.$ or TMS). All melting points reported are uncorrected. Contact angle (CA) measurements for both water and hexadecane on a surface were performed according to procedures in the manufacturer's manual using a Ramé-Hart Standard Automated Goniometer Model 200 employing DROP image standard software and equipped with an automated dispensing system with a $250 \mu \mathrm{L}$ syringe and an illuminated specimen stage assembly. $2 \mathrm{H}, 2 \mathrm{H}, 3 \mathrm{H}, 3 \mathrm{H}$-perfluorononoyl chloride, $2 \mathrm{H}, 2 \mathrm{H}, 3 \mathrm{H}, 3 \mathrm{H}$ perfluoroheptanoyl chloride, $1 H, 1 H, 2 H, 2 H$-perfluorooctyl isocyanate and $1 H, 1 H, 2 H, 2 H$-perfluorohexyl isocyanate were synthesized from the corresponding perfluoroalkylethyl iodides [34].

\subsection{Synthesis of $2 H, 2 H, 3 H, 3 H$-Perfluorononoyl Chloride and $1 H, 1 H, 2 H, 2 H$-Perfluorooctyl Isocyanate}

A four-neck reactor fitted with a stirrer, thermocouple, nitrogen purge, addition funnel and jacketed condenser was purged with nitrogen and charged with magnesium turnings (4.86 g, $202.5 \mathrm{mmol})$ and anhydrous ether $(300 \mathrm{~mL})$. To this suspension was slowly added a solution of $1 \mathrm{H}, 1 \mathrm{H}, 2 \mathrm{H}, 2 \mathrm{H}$ perfluorooctyl iodide $(94.8 \mathrm{~g}, 200 \mathrm{mmol})$ in ether $(100 \mathrm{~mL})$ over a $2 \mathrm{~h}$ period. After the addition of about $10 \mathrm{~mL}$ of iodide, the mixture was heated to $35^{\circ} \mathrm{C}$, and when the reaction was initiated the rest of the iodide was added, keeping the temperature below $35^{\circ} \mathrm{C}$. The mixture was stirred for $2 \mathrm{~h}$ at room temperature (RT) and cooled to $5-10^{\circ} \mathrm{C}$. Excess dry ice $(\sim 15 \mathrm{~g})$ was added in small portions with 
constant stirring. After 15 min, the mixture was quenched with $\mathrm{H}_{2} \mathrm{SO}_{4}(25 \%)$ and the ether phase was separated. The aqueous phase was extracted with ether $(2 \times 100 \mathrm{~mL})$. Combined ether phases were washed with $\mathrm{NaOH}$ solution $(25 \%, 3 \times 75 \mathrm{~mL})$. The combined alkaline phases were heated at $100{ }^{\circ} \mathrm{C}$ for 10 min and neutralized using $\mathrm{H}_{2} \mathrm{SO}_{4}(50 \%)$ after cooling to ice temperature. The precipitate formed was filtered and washed with water to obtain $2 \mathrm{H}, 2 \mathrm{H}, 3 \mathrm{H}, 3 \mathrm{H}$-perfluorononoic acid as a white crystalline solid (56.5 g, $14.1 \mathrm{mmol}, 72 \%)$. mp. $61-63{ }^{\circ} \mathrm{C}$.

The $2 \mathrm{H}, 2 \mathrm{H}, 3 \mathrm{H}, 3 \mathrm{H}$-perfluorononoic acid $(44.4 \mathrm{~g}, 113.2 \mathrm{mmol})$ was heated with $\mathrm{PCl}_{5}(35.4 \mathrm{~g}$, $169.8 \mathrm{mmol}$ ) at $110^{\circ} \mathrm{C}$ for $3 \mathrm{~h}$ and the resulting mixture fractionally distilled to obtain $2 \mathrm{H}, 2 \mathrm{H}, 3 \mathrm{H}, 3 \mathrm{H}$ perfluorononoyl chloride as a colorless liquid (44.5 g, $107.5 \mathrm{mmol}, 95 \%)$. bp. $129{ }^{\circ} \mathrm{C} @ 85 \mathrm{~mm} \mathrm{Hg}$.

A 3-necked flask fitted with a stirrer, an addition funnel and a condenser with $\mathrm{N}_{2}$ purge was charged with freshly distilled $2 \mathrm{H}, 2 \mathrm{H}, 3 \mathrm{H}, 3 \mathrm{H}$-perfluorononoyl chloride (16.26 g, $39.6 \mathrm{mmol}$ ). Trimethylsilyl azide $\left(\mathrm{TMSN}_{3}, 4.57 \mathrm{~g}, 39.61 \mathrm{~g}\right.$ ) was added dropwise via the addition funnel, keeping the temperature about $5-10{ }^{\circ} \mathrm{C}$. The mixture was then stirred at $10{ }^{\circ} \mathrm{C}$ for $30 \mathrm{~min}$ and heated at $75{ }^{\circ} \mathrm{C}$ for $1.5 \mathrm{~h}$. After the reaction was complete (GC analysis), trimethylsilyl chloride was distilled off at atmospheric pressure and then the product was fractionally distilled under vacuum to produce $1 \mathrm{H}, 1 \mathrm{H}, 2 \mathrm{H}, 2 \mathrm{H}$ perfluorooctyl isocyanate as a colorless liquid (11.9 g, $30.6 \mathrm{mmol}, 77 \%) \mathrm{bp} .100-103{ }^{\circ} \mathrm{C} @ 30 \mathrm{~mm} \mathrm{Hg}$.

\subsection{Synthesis of $2 H, 2 H, 3 H, 3 H$-Perfluoroheptanoyl Chloride and $1 H, 1 H, 2 H, 2 H$-Perfluorohexyl} Isocyanate

Using a similar procedure as described above, reaction of $1 H, 1 H, 2 H, 2 H$-perfluorohexyl iodide (74.8 g, $200 \mathrm{mmol})$ with $\mathrm{Mg}(4.86 \mathrm{~g}, 202.5 \mathrm{mmol})$ and dry-ice $(\sim 15 \mathrm{~g})$ produced $2 \mathrm{H}, 2 \mathrm{H}, 3 \mathrm{H}, 3 \mathrm{H}$ perfluoroheptanoic acid as a white crystalline solid (43 g, $145.7 \mathrm{mmol})$. Reaction of $2 \mathrm{H}, 2 \mathrm{H}, 3 \mathrm{H}, 3 \mathrm{H}$ perfluoroheptanoic acid $\left(30.0 \mathrm{~g}, 102.7 \mathrm{mmol}, \mathrm{mp} .44{ }^{\circ} \mathrm{C}\right)$ with $\mathrm{PCl}_{5}$, followed by distillation, produced $2 \mathrm{H}, 2 \mathrm{H}, 3 \mathrm{H}, 3 \mathrm{H}$-perfluoroheptanoyl chloride as a colorless liquid $\left(18.0 \mathrm{~g}, 61.8 \mathrm{mmol}, 60 \%\right.$, bp. $27{ }^{\circ} \mathrm{C} @$ $3 \mathrm{~mm} \mathrm{Hg}$. The $2 H, 2 H, 3 H, 3 H$-perfluoroheptanoyl chloride (4.30 g, $10.5 \mathrm{mmol})$ upon reaction with $\mathrm{TMSN}_{3}(1.45 \mathrm{~g}, 12.6 \mathrm{mmol})$, followed by fractional distillation, produced $1 \mathrm{H}, 1 \mathrm{H}, 2 \mathrm{H}, 2 \mathrm{H}$-perfluorohexyl isocyanate as a colorless liquid (2.5 g, $6.42 \mathrm{mmol}, 61 \%)$. bp.101-103 ${ }^{\circ} \mathrm{C} @ 50 \mathrm{~mm} \mathrm{Hg}$.

\subsection{Synthesis of $\mathbf{1}$}

A mixture of dichloromethane $(350 \mathrm{~mL}), \quad N$-Boc- $\beta$-alanine $(8.50 \mathrm{~g}, 45.0 \mathrm{mmol})$, 1-[3-(dimethylamino)propyl]-3-ethylcarbodiimide hydrochloride (EDCl) $(8.6 \mathrm{~g}, 45.0 \mathrm{mmol})$ and 4-(dimethylamino)pyridine $(5.49 \mathrm{~g}, 45.0 \mathrm{mmol}$ ) were stirred for $10 \mathrm{~min}$ at RT, followed by addition of octylamine ( $5.71 \mathrm{~g}, 45.0 \mathrm{mmol})$. The mixture was stirred for $12 \mathrm{~h}$ at RT. The mixture was washed with $5 \% \mathrm{HCl}(2 \times 100 \mathrm{~mL})$, saturated $\mathrm{NaHCO}_{3}$ solution $(1 \times 100 \mathrm{~mL})$ and brine $(1 \times 50 \mathrm{~mL})$. The resulting organic layer was dried $\left(\mathrm{MgSO}_{4}\right)$ and concentrated to provide $N$-Boc- $\beta$-ala-octylamide (1) as a white crystalline solid (11.6 g, $38.6 \mathrm{mmol}, 86 \%$ ): mp. 67.5-68.4 ${ }^{\circ} \mathrm{C} ;{ }^{1} \mathrm{H}$ NMR (methanol- $d 4$ ): $\delta 3.18$ (t, J = 7.0 Hz, 2H), $2.90(\mathrm{t}, J=6.5 \mathrm{~Hz}, 2 \mathrm{H}), 2.35(\mathrm{t}, \mathrm{J}=6.5 \mathrm{~Hz}, 2 \mathrm{H}), 1.51$ (quintet, $J=7.0 \mathrm{~Hz}, 2 \mathrm{H}$ ), $1.34(\mathrm{~m}, 10 \mathrm{H}), 0.93$ (t, distorted, $J=6.5 \mathrm{~Hz}, 3 \mathrm{H})$ : 


\subsection{Synthesis of 2}

A suspension of $1(10.5 \mathrm{~g}, 35.0 \mathrm{mmol})$ in dichloromethane $(40 \mathrm{~mL})$ was stirred with trifluoroacetic acid (TFA) (31.9 g, $280.0 \mathrm{mmol})$ at RT for $3 \mathrm{~h}$. Chloroform $(100 \mathrm{~mL})$ was added and the bulk of the solvents and TFA evaporated under vacuum. The resulting trifluoroacetate salt was suspended in dichloromethane $(50 \mathrm{~mL})$ and stirred with dropwise addition of saturated $\mathrm{NaHCO}_{3}$ solution $(200 \mathrm{~mL})$ and the solid formed was filtered. The residue was washed several times with cold water and dried under vacuum to provide compound 2 as a white solid (6.5 g, 32.5 mmol, 93\%): mp. $142-143.5{ }^{\circ} \mathrm{C} .{ }^{1} \mathrm{H}$ NMR (methanol- $d 4): \delta 3.46$ (t, $J=7.2 \mathrm{~Hz}, 2 \mathrm{H}), 3.17$ (t, $J=7.0 \mathrm{~Hz}, 2 \mathrm{H}), 2.58(\mathrm{~m}, 2 \mathrm{H}), 2.40(\mathrm{t}, J=6.5 \mathrm{~Hz}$, $4 \mathrm{H}$ ), 1.50 (quintet, $J=7.0 \mathrm{~Hz}, 2 \mathrm{H}), 1.34(\mathrm{~m}, 10 \mathrm{H}), 0.93$ (t, distorted, $3 \mathrm{H}$ ): ${ }^{19} \mathrm{~F}$ NMR (methanol d4): $\delta$ $-82.8(\mathrm{~m}, 3 \mathrm{~F}),-115.9(\mathrm{~m}, 2 \mathrm{~F}),-123.3(\mathrm{~s}, 2 \mathrm{~F}),-124.3(\mathrm{~s}, 2 \mathrm{~F}),-125.0(\mathrm{~m}, 2 \mathrm{~F}),-127.7(\mathrm{~m}, 2 \mathrm{~F})$.

\subsection{Synthesis of $\mathbf{3}$}

Using a similar procedure as described for the synthesis of compound 1, reaction of $N$-Boc-Laspartic acid (10.0 g, $43.1 \mathrm{mmol})$ with $n$-octylamine (11.1 g, $86.2 \mathrm{mmol}) 1$-[3-(dimethylamino)propyl]3-ethylcarbodiimide hydrochloride $(\mathrm{EDCl})$ (16.5 g, $86.2 \mathrm{mmol})$ and 4-(dimethylamino)pyridine $(5.25 \mathrm{~g}, 43.1 \mathrm{mmol})$ in dichloromethane $(300 \mathrm{~mL})$ provided $N$-Boc-L-Asp-dioctylamide 3 (18.8 $\mathrm{g}$, $41.3 \mathrm{mmol}, 92 \%)$. mp. $112-113{ }^{\circ} \mathrm{C}$.

\subsection{Synthesis of $\mathbf{4}$}

Using a similar procedure as described for the synthesis of compound 2, reaction of 3 (18.6 g, $41.0 \mathrm{mmol})$ with trifluoroacetic acid $(46.7 \mathrm{~g}, 410 \mathrm{mmol})$ in dichloromethane $(50.0 \mathrm{~mL})$ provided compound 4 (13.4 g, 96\%) as a white solid. mp. 120.5-122 ${ }^{\circ} \mathrm{C} .{ }^{1} \mathrm{H}$ NMR (acetone-d6) $\delta 7.14$ (bs, 2H), $4.35(\mathrm{dd}, J=8.8,4.6 \mathrm{~Hz}, 1 \mathrm{H}), 3.18(\mathrm{~m}, 4 \mathrm{H}), 2.66(\mathrm{dd}, J=14.0,4.0 \mathrm{~Hz}, 1 \mathrm{H}), 2.35(\mathrm{dd}, J=14.0,8.0 \mathrm{~Hz}$, 1H), 1.90 (bm, 2H), 1.50 (m, 4H), $1.31(\mathrm{~m}, 10 \mathrm{H}), 0.90$ (t, $J=6.8 \mathrm{~Hz}, 3 \mathrm{H})$.

\subsection{Synthesis of $\mathbf{5 a}$}

To a mixture of $2(1.0 \mathrm{~g}, 5.0 \mathrm{mmol})$, dichloromethane $(20 \mathrm{~mL})$ and triethylamine $(0.526 \mathrm{~g}$, $0.52 \mathrm{mmol}$ ) under a $\mathrm{N}_{2}$ purge was added $2 \mathrm{H}, 2 \mathrm{H}, 3 \mathrm{H}, 3 \mathrm{H}$-perfluorononoyl chloride $(2.13 \mathrm{~g}, 5.2 \mathrm{mmol})$ and the mixture stirred for $5 \mathrm{~h}$ at RT. The bulk of the dichloromethane was evaporated and the solid obtained was taken in ethyl acetate $(60 \mathrm{~mL})$ and then washed with $2 \% \mathrm{HCl}(2 \times 30 \mathrm{~mL})$, water $(1 \times 30 \mathrm{~mL})$ and brine $(1 \times 30 \mathrm{~mL})$. The resulting organic layer was dried $\left(\mathrm{MgSO}_{4}\right)$ and concentrated to provide 5a as a white solid (2.46 g, $4.29 \mathrm{mmol}, 86 \%):{ }^{1} \mathrm{H}$ NMR (methanol- $\left.d 4\right): \delta 3.46(\mathrm{t}, J=7.2 \mathrm{~Hz}$, $2 \mathrm{H}), 3.17(\mathrm{t}, J=7.0 \mathrm{~Hz}, 2 \mathrm{H}), 2.58(\mathrm{~m}, 2 \mathrm{H}), 2.40(\mathrm{t}, J=6.5 \mathrm{~Hz}, 4 \mathrm{H}), 1.50$ (quintet, $J=7.0 \mathrm{~Hz}, 2 \mathrm{H}$ ), $1.34(\mathrm{~m}, 10 \mathrm{H}), 0.93(\mathrm{t}$, distorted, $3 \mathrm{H}):{ }^{19} \mathrm{~F}$ NMR (methanol- $\left.d 4\right): \delta-82.8(\mathrm{~m}, 3 \mathrm{~F}),-115.9(\mathrm{~m}, 2 \mathrm{~F})$, -123.3 (s, 2F), -124.3 (s, 2F), -125.0 (m, 2F), -127.7 (m, 2F).

\subsection{Synthesis of $\mathbf{5 b}$}

Using a similar procedure as described for the synthesis of compound 5a, reaction of 2 (1.37 g, $6.85 \mathrm{mmol})$ with $2 \mathrm{H}, 2 \mathrm{H}, 3 \mathrm{H}, 3 \mathrm{H}$-perfluoroheptanoyl chloride $(2.13 \mathrm{~g}, 6.85 \mathrm{mmol})$ and triethylamine 
(0.69 g, $6.85 \mathrm{mmol})$ provided $\mathbf{5 b}$ as a white solid (2.8 g, $5.90 \mathrm{mmol}, 86 \%): \mathrm{mp} .99-102{ }^{\circ} \mathrm{C} ;{ }^{1} \mathrm{H} \mathrm{NMR}$ $\left(\mathrm{CDCl}_{3}\right): \delta 6.78(\mathrm{bs}, 1 \mathrm{H}), 5.81(\mathrm{bs}, 1 \mathrm{H}), 3.56(\mathrm{q}, J=6.4 \mathrm{~Hz}, 2 \mathrm{H}), 3.25(\mathrm{q}, J=7.2 \mathrm{~Hz}, 2 \mathrm{H}), 2.47$ (m, $2 \mathrm{H}), 2.43(\mathrm{t}, J=5.6 \mathrm{~Hz}, 4 \mathrm{H}), 1.51$ (quintet, $J=7.0 \mathrm{~Hz}, 2 \mathrm{H}), 1.30(\mathrm{~m}, 10 \mathrm{H}), 0.901(\mathrm{t}, J=7.5 \mathrm{~Hz}, 3 \mathrm{H})$;

${ }^{19} \mathrm{~F} \mathrm{NMR}\left(\mathrm{CDCl}_{3}\right): \delta-81.6(\mathrm{~m}, 3 \mathrm{~F}),-115.3(\mathrm{~m}, 2 \mathrm{~F}),-125.0(\mathrm{~m}, 2 \mathrm{~F}),-126.6(\mathrm{~m}, 2 \mathrm{~F})$.

\subsection{Synthesis of $\mathbf{5 c}$}

Using a similar procedure as described for the synthesis of compound 5a, reaction of 2 (1.37 g, $6.85 \mathrm{mmol})$ with pentafluorobenzoyl chloride $(1.57 \mathrm{~g}, 6.85 \mathrm{mmol})$ and triethylamine $(0.69 \mathrm{~g}$, $6.85 \mathrm{mmol})$ provided $\mathbf{5 c}$ as a white solid $(2.2 \mathrm{~g}, 5.58 \mathrm{mmol}, 82 \%): \mathrm{mp} .154-155{ }^{\circ} \mathrm{C}$; ${ }^{1} \mathrm{H}$ NMR (methanol-d4): $\delta 3.65$ (t, $J=6.8 \mathrm{~Hz}, 2 \mathrm{H}), 3.19$ (t, $J=7.2 \mathrm{~Hz}, 2 \mathrm{H}), 2.53(\mathrm{t}, J=5.6 \mathrm{~Hz}, 2 \mathrm{H}), 1.52$ (quintet, $J=7.0 \mathrm{~Hz}, 2 \mathrm{H}), 1.34(\mathrm{~m}, 10 \mathrm{H}), 0.92$ (t, distorted, $3 \mathrm{H}) ;{ }^{19} \mathrm{~F}$ NMR (methanol- $\left.d 4\right): \delta-144.3$ $(\mathrm{m}, 2 \mathrm{~F}),-155.8(\mathrm{~m}, 1 \mathrm{~F}),-164.3(\mathrm{~m}, 2 \mathrm{~F})$.

\subsection{Synthesis of $\mathbf{6 a}$}

Using a similar procedure as described for the synthesis of compound 5a, reaction of 4 (1.775 g, $5.0 \mathrm{mmol})$ with $2 \mathrm{H}, 2 \mathrm{H}, 3 \mathrm{H}, 3 \mathrm{H}$-perfluorononoyl chloride $(0.438 \mathrm{~g}, 2.7 \mathrm{mmol})$ provided $\mathbf{6 a}$ as a white solid (2.8 g, 77\%): mp. 178-180 ${ }^{\circ} \mathrm{C} ;{ }^{1} \mathrm{H}$ NMR (acetone-d6) $\delta 7.52$ (bs, 1H), 7.17 (bs, 1H), 7.06 (bs, $1 \mathrm{H}), 4.55(\mathrm{~m}, 1 \mathrm{H}), 3.02(\mathrm{bs}, 4 \mathrm{H}), 2.55-2.40(\mathrm{~m}, 6 \mathrm{H}), 1.27$ (m, 4H), 1.16 (bs, $20 \mathrm{H}), 0.75(\mathrm{bs}, 6 \mathrm{H}) ;{ }^{19} \mathrm{~F}$ NMR (acetone-d6): $\delta-82.1(\mathrm{~s}, 3 \mathrm{~F}),-115.2(\mathrm{~s}, 2 \mathrm{~F}),-122.8(\mathrm{~s}, 2 \mathrm{~F}),-123.8(\mathrm{~s}, 2 \mathrm{~F}),-124.4(\mathrm{~s}, 2 \mathrm{~F})$, $-127.1(\mathrm{~s}, 2 \mathrm{~F})$.

\subsection{Synthesis of $\mathbf{6 b}$}

Using a similar procedure as described for the synthesis of compound 5a, reaction of 4 (1.77 $\mathrm{g}$, $5.0 \mathrm{mmol}$ ) with $2 \mathrm{H}, 2 \mathrm{H}, 3 \mathrm{H}, 3 \mathrm{H}$-perfluoroheptanoyl chloride (1.55 g, $5.0 \mathrm{mmol})$ provided $\mathbf{6 b}$ as a white solid (1.92 g, 61\%): mp. 163.5-165.5 ${ }^{\circ} \mathrm{C} ;{ }^{1} \mathrm{H}$ NMR (DMF-d7): $\delta 8.80$ (bs, 1H), 8.0 (bs, 1H), 7.96 (bs, $1 \mathrm{H}), 4.91(\mathrm{q}, J=6.0 \mathrm{~Hz}, 1 \mathrm{H}), 3.32(\mathrm{~m}, 4 \mathrm{H}), 2.87-2.70(\mathrm{~m}, 4 \mathrm{H}), 1.63(\mathrm{~m}, 4 \mathrm{H}), 1.45$ (bs, $20 \mathrm{H}), 0.75$ (t, distorted, $6 \mathrm{H}) ;{ }^{19} \mathrm{~F}$ NMR (DMF-d7): $\delta-81.8(\mathrm{~m}, 3 \mathrm{~F}),-114.9(\mathrm{~m}, 2 \mathrm{~F}),-125.9(\mathrm{~m}, 2 \mathrm{~F}),-126.6(\mathrm{~m}, 2 \mathrm{~F})$.

\subsection{Synthesis of $\mathbf{6 c}$}

Using a similar procedure as described for the synthesis of compound 5a, reaction of 4 (1.77 $\mathrm{g}$, $5.0 \mathrm{mmol})$ with pentafluorobenzoyl chloride $(1.15 \mathrm{~g}, 5.0 \mathrm{mmol})$ provided $\mathbf{6 c}$ as a white solid $(1.8 \mathrm{~g}$, 66\%): mp. 172-183.5 ${ }^{\circ} \mathrm{C} ;{ }^{1} \mathrm{H}$ NMR (DMF-d7): $\delta 9.11(\mathrm{~d}, J=8.0 \mathrm{~Hz}, 1 \mathrm{H}), 7.88(\mathrm{~m} \mathrm{2H}), 4.96$ (q, $J=6.8 \mathrm{~Hz}, 1 \mathrm{H}), 3.19(\mathrm{~m}, 4 \mathrm{H}), 2.78-2.70(\mathrm{~m}, 2 \mathrm{H}) .1 .48(\mathrm{~m}, 4 \mathrm{H}), 1.29$ (bs, $20 \mathrm{H}), 0.89$ (t, distorted, $6 \mathrm{H}) ;{ }^{19} \mathrm{~F}$ NMR (DMF- $\left.d 7\right): \delta-143.0(\mathrm{~m}, 2 \mathrm{~F}),-155.5(\mathrm{t}, J=21.6 \mathrm{~Hz}, 1 \mathrm{~F}),-163.9(\mathrm{~m}, 2 \mathrm{~F})$.

\subsection{Synthesis of $7 \mathbf{a}$}

To a mixture of $2(0.822 \mathrm{~g}, 4.11 \mathrm{mmol})$, dichloromethane $(20 \mathrm{~mL})$ and triethylamine $(0.02 \mathrm{~g}$, $0.2 \mathrm{mmol}$ ) under a $\mathrm{N}_{2}$ purge was added $1 H, 1 H, 2 H, 2 H$-perfluorooctyl ioscyanate $(1.75 \mathrm{~g}, 4.52 \mathrm{mmol})$, and the mixture stirred for $5 \mathrm{~h}$ at RT. The solid product was filtered and washed with cold hexanes $(2 \times 5 \mathrm{~mL})$ to provide $7 \mathbf{a}$ as a white solid $(1.8 \mathrm{~g}, 3.05 \mathrm{mmol}, 74 \%): \mathrm{mp} .132-136{ }^{\circ} \mathrm{C} ;{ }^{1} \mathrm{H} \mathrm{NMR}$ 
(methanol-d4): $\delta 3.46(\mathrm{t}, J=7.2 \mathrm{~Hz}, 2 \mathrm{H}), 3.39(\mathrm{t}, J=6.4 \mathrm{~Hz}, 2 \mathrm{H}), 2.94(\mathrm{~m}, 2 \mathrm{H}), 2.60(\mathrm{t}, J=6.0 \mathrm{~Hz}$, 2H), $2.39(\mathrm{~m}, 2 \mathrm{H}), 1.53(\mathrm{~m}, 2 \mathrm{H}), 1.34$ (bs, 10H), 0.92 (t, distorted, 3H); ${ }^{19} \mathrm{~F}$ NMR (methanol-d4): $\delta$ $-82.8(\mathrm{~m}, 3 \mathrm{~F}),-115.6(\mathrm{~m}, 2 \mathrm{~F}),-123.2(\mathrm{~s}, 2 \mathrm{~F}),-124.2(\mathrm{~s}, 2 \mathrm{~F}),-125.0(\mathrm{~m}, 2 \mathrm{~F}),-127.7$ (m, 2F).

\subsection{Synthesis of $\mathbf{7 b}$}

Using a similar procedure as described for the synthesis of compound 7a, reaction of $2(0.629 \mathrm{~g}$, $3.14 \mathrm{mmol}$ ) with $1 \mathrm{H}, 1 \mathrm{H}, 2 \mathrm{H}, 2 \mathrm{H}$-perfluorohexyl isocyanate $(1.2 \mathrm{~g}, 4.15 \mathrm{mmol})$ provided $7 \mathbf{b}(1.08 \mathrm{~g}$, 67\%): ${ }^{1} \mathrm{H}$ NMR (methanol-d4): $\delta 3.46(\mathrm{t}, J=7.2 \mathrm{~Hz}, 2 \mathrm{H}), 3.39(\mathrm{t}, J=6.4 \mathrm{~Hz}, 2 \mathrm{H}), 3.19(\mathrm{~m}, 2 \mathrm{H}), 2.61$ $(\mathrm{t}, J=6.0 \mathrm{~Hz}, 2 \mathrm{H}), 2.39(\mathrm{~m}, 2 \mathrm{H}), 1.53(\mathrm{~m}, 2 \mathrm{H}), 1.34(\mathrm{bs}, 10 \mathrm{H}), 0.92(\mathrm{t}$, distorted, $3 \mathrm{H}) ;{ }^{19} \mathrm{~F}$ NMR (methanol- $d 4)$ : $\delta-83.0(\mathrm{~m}, 3 \mathrm{~F}),-115.8(\mathrm{~m}, 2 \mathrm{~F}),-126.1(\mathrm{~m}, 2 \mathrm{~F}),-127.6(\mathrm{~m}, 2 \mathrm{~F})$.

\subsection{Synthesis of $7 \mathrm{c}$}

Using a similar procedure as described for the synthesis of compound 7a, reaction of $2(1.0 \mathrm{~g}$, $5.0 \mathrm{mmol})$ with pentafluorophenyl isocyanate $(1.04 \mathrm{~g}, 5.0 \mathrm{mmol})$ provided $7 \mathrm{c}$ as a white solid $(1.72 \mathrm{~g}$, 84\%): mp. $175-176{ }^{\circ} \mathrm{C} ;{ }^{1} \mathrm{H}$ NMR (methanol-d4): $\delta 3.48$ (t, $\left.J=6.8 \mathrm{~Hz}, 2 \mathrm{H}\right), 3.20(\mathrm{t}, J=7.2 \mathrm{~Hz}, 2 \mathrm{H}$ ), $2.45(\mathrm{t}, J=5.6 \mathrm{~Hz}, 2 \mathrm{H}), 1.52$ (quintet, $J=7.0 \mathrm{~Hz}, 2 \mathrm{H}), 1.33(\mathrm{~m}, 10 \mathrm{H}), 0.91(\mathrm{t}, J=7.5 \mathrm{~Hz}, 3 \mathrm{H}) ;{ }^{19} \mathrm{~F}$ NMR (methanol-d4): $\delta-149.4(\mathrm{~m}, 2 \mathrm{~F}),-162.8(\mathrm{t}, J=20.6 \mathrm{~Hz}, 1 \mathrm{~F}),-167.3(\mathrm{~m}, 2 \mathrm{~F})$.

\subsection{Synthesis of $\mathbf{8 a}$}

Using a similar procedure as described for the synthesis of compound 7a, reaction of 4 ( $0.912 \mathrm{~g}$, $2.57 \mathrm{mmol})$ with $1 \mathrm{H}, 1 \mathrm{H}, 2 \mathrm{H}, 2 \mathrm{H}$-perfluorooctyl isocyanate $(1.16 \mathrm{~g}, 3.0 \mathrm{mmol})$ provided $\mathbf{8 a}$ as a white solid (1.1 g, $1.47 \mathrm{mmol}, 58 \%):{ }^{1} \mathrm{H}$ NMR (methanol-d4): $\delta 4.54(\mathrm{t}, J=6.8 \mathrm{~Hz}, 1 \mathrm{H}), 3.46(\mathrm{t}, J=6.8 \mathrm{~Hz}$, 2H), $3.21(\mathrm{~m}, 4 \mathrm{H}), 2.60(\mathrm{~m}, 2 \mathrm{H}), 2.40(\mathrm{~m}, 2 \mathrm{H}), 1.53(\mathrm{~m}, 4 \mathrm{H}), 1.33$ (bs, 20H), 0.922 (t, distorted, 6H);

${ }^{19} \mathrm{~F}$ NMR (methanol-d4): $\delta-82.8(\mathrm{~m}, 3 \mathrm{~F}),-115.6(\mathrm{~s}, 2 \mathrm{~F}),-123.2(\mathrm{~s}, 2 \mathrm{~F}),-124.2$ (s, 2F), -125.0 $(\mathrm{s}, 2 \mathrm{~F}),-127.6(\mathrm{~m}, 2 \mathrm{~F})$.

\subsection{Synthesis of $\mathbf{8 b}$}

Using a similar procedure as described for the synthesis of compound 7a, reaction of $4(0.79 \mathrm{~g}$, $2.24 \mathrm{mmol})$ with $1 \mathrm{H}, 1 \mathrm{H}, 2 \mathrm{H}, 2 \mathrm{H}$-perfluorohexyl isocyanate $(1.0 \mathrm{~g}, 3.26 \mathrm{mmol})$ provided $\mathbf{8 b}$ as a white solid (1.51 g, 71\%) ${ }^{1} \mathrm{H}$ NMR (methanol-d4): $\delta 4.54(\mathrm{t}, J=6.8 \mathrm{~Hz}, 1 \mathrm{H}), 3.46(\mathrm{t}, J=6.8 \mathrm{~Hz}, 2 \mathrm{H}), 3.22$ $(\mathrm{m}, 4 \mathrm{H}), 2.61(\mathrm{~m}, 2 \mathrm{H}), 2.41(\mathrm{~m}, 2 \mathrm{H}), 1.51(\mathrm{~m}, 4 \mathrm{H}), 1.33(\mathrm{bs}, 20 \mathrm{H}), 0.922(\mathrm{t}$, distorted, $6 \mathrm{H}) ;{ }^{19} \mathrm{~F}$ NMR (methanol- $d 4)$ : $\delta-83.0(\mathrm{~m}, 3 \mathrm{~F}),-115.8(\mathrm{~m}, 2 \mathrm{~F}),-126.0(\mathrm{~m}, 2 \mathrm{~F}),-127.6(\mathrm{~m}, 2 \mathrm{~F})$.

\subsection{Synthesis of $\mathbf{8 c}$}

Using a similar procedure as described for the synthesis of compound 7a, reaction of 4 (1.77 $\mathrm{g}$, $5.0 \mathrm{mmol})$ with pentafluorophenyl isocyanate $(1.04 \mathrm{~g}, 5.0 \mathrm{mmol})$ provided $8 \mathrm{c}$ as a white solid $(2.0 \mathrm{~g}$, $3.45 \mathrm{mmol}, 71 \%):{ }^{1} \mathrm{H}$ NMR (DMF-d7, $\left.100{ }^{\circ} \mathrm{C}\right): \delta 8.74(\mathrm{~m}, 1 \mathrm{H}), 7.57$ (bs, 2H), $7.17(\mathrm{bs}, 1 \mathrm{H}), 4.74(\mathrm{q}$, $J=6.8 \mathrm{~Hz}, 1 \mathrm{H}), 3.35(\mathrm{~m}, 4 \mathrm{H}), 2.86(\mathrm{dd}, J=15.2,6.0 \mathrm{~Hz}, 1 \mathrm{H}), 2.78(\mathrm{dd}, J=15.2,6.0 \mathrm{~Hz}, 1 \mathrm{H}), 1.65$ $(\mathrm{m}, 4 \mathrm{H}), 1.47(\mathrm{bs}, 20 \mathrm{H}), 1.03\left(\mathrm{t}\right.$, distorted, 6H); ${ }^{19} \mathrm{~F}$ NMR (DMF-d7): $\delta-147.8(\mathrm{~m}, 2 \mathrm{~F}),-163.2$ $(\mathrm{t}, J=22.0 \mathrm{~Hz}, 1 \mathrm{~F}),-168.3(\mathrm{~m}, 2 \mathrm{~F})$. 


\subsection{Synthesis of 9}

Using a similar procedure as described for the synthesis of 1, reaction of $N$-Boc- $\beta$-alanine ( $8.5 \mathrm{~g}$, $45.0 \mathrm{mmol})$ with $n$-octanol (5.85 g, $45.0 \mathrm{mmol}), 1$-[3-(dimethylamino)propyl]-3-ethylcarbodiimide hydrochloride $(8.62 \mathrm{~g}, 45.0 \mathrm{mmol})$ and 4-(dimethylamino)pyridine $(5.49 \mathrm{~g}, 45.0 \mathrm{mmol})$ in dichloromethane $(350 \mathrm{~mL})$ provided $n$-octyl- $N$-Boc- $\beta$-alanine $(12.75 \mathrm{~g}, 42.3 \mathrm{mmol}, 94 \%)$. Using a similar procedure as described for the synthesis of $2, n$-octyl- $N$-Boc- $\beta$-alanine $(12.0 \mathrm{~g}, 39.87 \mathrm{mmol})$ was deprotected with trifluoroacetic acid to obtain $n$-octyl- $\beta$-alanine $(6.8 \mathrm{~g}, 33.8 \mathrm{mmol}, 85 \%)$. Reaction of $n$-octyl- $\beta$-alanine $(0.573 \mathrm{~g}, 2.85 \mathrm{mmol})$ with $1 H, 1 H, 2 H, 2 H$-perfluorooctyl isocyanate (1.1 g, $2.85 \mathrm{mmol})$ provided 9 as a pale yellow oil $(1.95 \mathrm{~g}, 62 \%):{ }^{1} \mathrm{H} \mathrm{NMR}\left(\mathrm{CDCl}_{3}\right): \delta 4.06(\mathrm{t}, J=7.2 \mathrm{~Hz}$, $2 \mathrm{H}), 3.53(\mathrm{t}, J=6.4 \mathrm{~Hz}, 2 \mathrm{H}), 3.26(\mathrm{~m}, 2 \mathrm{H}), 2.30(\mathrm{~m}, 2 \mathrm{H}), 1.55(\mathrm{~m}, 2 \mathrm{H}), 1.20(\mathrm{bs}, 10 \mathrm{H}), 0.81$ (t, distorted, $3 \mathrm{H}) ;{ }^{19} \mathrm{~F}$ NMR $\left(\mathrm{CDCl}_{3}\right): \delta-81.2(\mathrm{~m}, 3 \mathrm{~F}),-114.4(\mathrm{~m}, 2 \mathrm{~F}),-122.2(\mathrm{~s}, 2 \mathrm{~F}),-123.2(\mathrm{~m}, 2 \mathrm{~F})$, $-124.0(\mathrm{~m}, 2 \mathrm{~F}),-126.5(\mathrm{~m}, 2 \mathrm{~F})$.

\subsection{Synthesis of $\mathbf{1 0}$}

Using a similar procedure as described for the synthesis of $\mathbf{1}$, reaction of $N$-Boc-L-aspartic acid $(10.0 \mathrm{~g}, 43.1 \mathrm{mmol})$ with $n$-octanol $(11.2 \mathrm{~g}, 86.2 \mathrm{mmol})$ provided $N$-Boc-dioctylaspartate as an oil (19.0 g, $41.5 \mathrm{mmol}, 96 \%):{ }^{1} \mathrm{H}$ NMR $\left(\mathrm{CDCl}_{3}\right): \delta 5.49(\mathrm{~d}, J=8 \mathrm{~Hz}, 1 \mathrm{H}), 4.53(\mathrm{~m}, 1 \mathrm{H}), 4.14(\mathrm{~m}, 2 \mathrm{H})$, $4.06(\mathrm{t}, J=7.2 \mathrm{~Hz}, 2 \mathrm{H}), 2.95(\mathrm{dd}, J=17.2,4.4 \mathrm{~Hz}, 1 \mathrm{H}), 2.78$ (dd, $J=17.8,5.2 \mathrm{~Hz}, 1 \mathrm{H}), 1.60$ (quintet, $J=7.2 \mathrm{~Hz}, 4 \mathrm{H}), 1.29$ (bm, 10H), 1.44 (s, 9H) $0.87 \mathrm{t}, J=7.8 \mathrm{~Hz}, 6 \mathrm{H})$.

Using a similar procedure as described for the synthesis of 2, $N$-Boc-dioctylaspartate $(19.0 \mathrm{~g}$, $41.5 \mathrm{mmol}$ ) was deprotected using trifluoroacetic acid to obtain dioctylaspartate as a viscous oil which solidified in the freezer $(12.1 \mathrm{~g}, 34.0 \mathrm{mmol}, 82 \%) .{ }^{1} \mathrm{H} \mathrm{NMR}\left(\mathrm{CDCl}_{3}\right): \delta 4.16(\mathrm{~m}, 4 \mathrm{H}), 3.82(\mathrm{dd}, J=7.2$, $5.3 \mathrm{~Hz}, 1 \mathrm{H}), 2.95$ (dd, $J=17.6,4.6 \mathrm{~Hz}, 1 \mathrm{H}), 2.71$ (dd, $J=17.6,4.5 \mathrm{~Hz}, 1 \mathrm{H}), 1.80$ (bs, 2H), 1.62 (m, $4 \mathrm{H}), 1.31(\mathrm{~m}, 10 \mathrm{H}), 0.90(\mathrm{t}, J=6.8 \mathrm{~Hz}, 6 \mathrm{H})$. Using a similar procedure as described for the synthesis of 7a, reaction of octyldiaspartate $(0.629 \mathrm{~g}, 3.14 \mathrm{mmol})$ with $1 H, 1 H, 2 H, 2 H$-perfluorohexyl isocyanate $(1.21 \mathrm{~g}, 4.21 \mathrm{mmol})$ provided 10 as an oil $(1.12 \mathrm{~g}, 67 \%)$ : $1 \mathrm{H} \mathrm{NMR}(\mathrm{CDCl} 3): \delta .5 .70$ (bs, $1 \mathrm{H}), 5.39$ (bs, $1 \mathrm{H}), 4.75(\mathrm{~m}, 1 \mathrm{H}), 4.15-3.4 .0(\mathrm{~m}, 4 \mathrm{H}), 3.55(\mathrm{t}, J=6.4 \mathrm{~Hz}, 2 \mathrm{H}), 2.97(\mathrm{dd}, J=16.8,4.8 \mathrm{~Hz}, 1 \mathrm{H}), 2.78$ $(\mathrm{dd}, J=16.8,4.8 \mathrm{~Hz}, 1 \mathrm{H}), 2.34(\mathrm{~m}, 2 \mathrm{H}), 1.60(\mathrm{~m}, 4 \mathrm{H}), 1.26(\mathrm{~m}, 16 \mathrm{H}), 0.93(\mathrm{t}, J=6.8 \mathrm{~Hz}, 6 \mathrm{H}) ; 19 \mathrm{~F}$ NMR (CDCl3): $\delta .-81.5(\mathrm{~m}, 3 \mathrm{~F}),-114.5(\mathrm{~m}, 2 \mathrm{~F}),-122.4(\mathrm{~s}, 2 \mathrm{~F}),-123.5(\mathrm{~s}, 2 \mathrm{~F}),-124.4(\mathrm{~s}, 2 \mathrm{~F})$, $-126.7(\mathrm{~m}, 2 \mathrm{~F})$.

\subsection{Gelation Experiments in Organic Solvents}

Typically, $1-4 \mathrm{mg} / \mathrm{mL}$ of a gelator in an organic solvent in a closed vial was heated in a reactor block equipped to heat multiple vials. The vials were heated to $5-10{ }^{\circ} \mathrm{C}$ below the boiling point of the solvent until a clear solution was obtained, and then were allowed to cool to RT either by switching off the heat or by transferring the vials to a constant temperature water bath kept at $25^{\circ} \mathrm{C}$. The state of the solution was evaluated after 2-12 h. Stability of the gel was tested by inverting the vial, and gel samples for which the whole mass remains a single phase (no phase separation between solid and liquid phase) are considered stable. 


\subsection{Gel-Impregnation of Nonwoven Supports in Organic Solvents}

Nonwoven fabrics $\left(\right.$ Tyvek $^{\circledR}$ polyethylene nonwoven fabric and Kolon ${ }^{\circledR}$ spun-bonded polyester fabric (about $3.0 \mathrm{~cm} \times 3.0 \mathrm{~cm}$ ) were immersed in a suspension of amide or urea-amide gelator (1-3 $\mathrm{mg} / \mathrm{mL})$ in organic solvent kept in closed reaction flask equipped with a stir bar and temperature controller. The mixtures were heated to $5^{\circ} \mathrm{C}$ below the boiling point of the solvent for $1-2 \mathrm{~h}$ until clear solutions formed. The flasks were then either rapidly cooled to RT by removing the oil bath or slowly cooled to RT by switching off the heat. Gel formation was usually observed in about $0.5-6 \mathrm{~h}$, and the gels were allowed to age for an additional $6 \mathrm{~h}$. The gelator-impregnated samples were removed and dried in a vacuum oven at RT overnight. The dried samples were weighed and used for contact angle measurements.

\section{Results and Discussion}

\subsection{Organogelators: Design and Synthesis}

Several new partially fluorinated organogelators were designed via careful manipulation of hydrogen bonding functionalities (urea and amide) and hydrophobic functionalities (fluorocarbon and hydrocarbon) in the molecule to promote gelation. Boc-protected amino acids, $N$-Boc- $\beta$-alanine and $N$-Boc-L-aspartic acid were chosen as the building blocks. First $N$-Boc- $\beta$-alanine and $N$-Boc-L-aspartic acid were transformed to the corresponding $N$-Boc-octylamides (1 and $\mathbf{3}$ ) by reacting with octylamine (Scheme 1). The resulting amides were deprotected to obtain the corresponding free amide-amine derivatives ( $\mathbf{2}$ and $\mathbf{4}$ ), the primary building blocks for partially fluorinated amide or urea-amide derivatives.

Scheme 1. Synthesis of 3-amino- $N$-octylpropanamide (2) L-asparticdioctylamide (4) building blocks from $N$-Boc- $\beta$-alanine and $N$-Boc-L-aspartic acid.
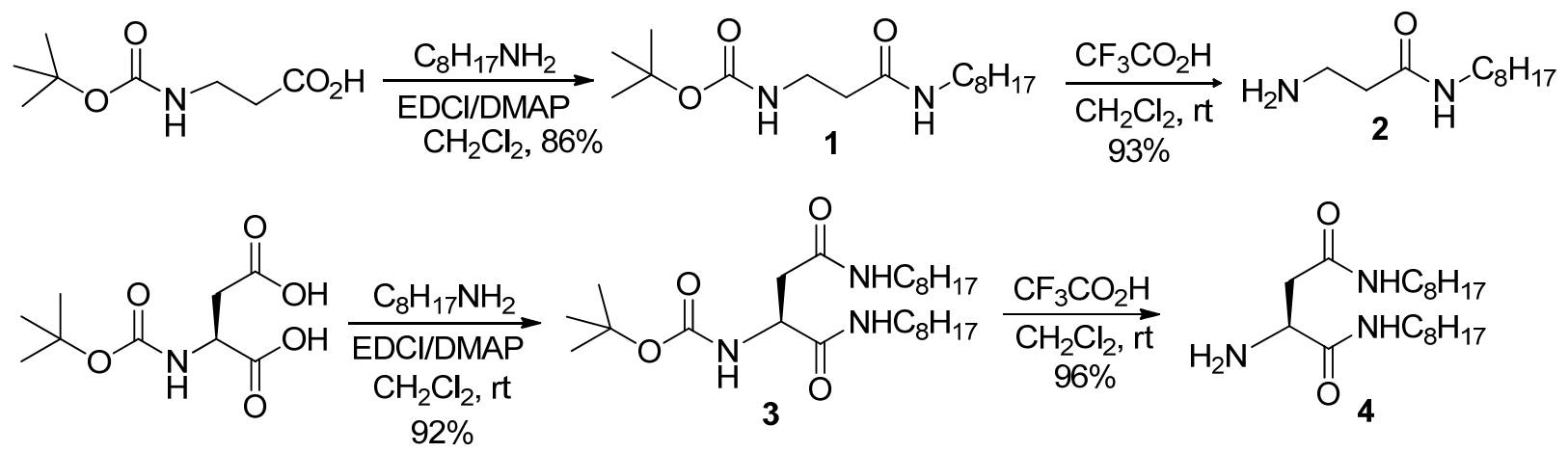

\subsubsection{Partially Fluorinated-bis- and Tris-Amides}

The amide-amine building blocks ( 2 and 4) were used for the preparation of partially fluorinated amide derivatives as shown in Scheme 2. Reaction of 2 with $2 H, 2 H, 3 H, 3 H$-perfluorononoyl chloride or $2 \mathrm{H}, 2 \mathrm{H}, 3 \mathrm{H}, 3 \mathrm{H}$-perfluoroheptanoylchloride provided the bis-amide derivatives $\mathbf{5 a}$ or $\mathbf{5 b}$, respectively, in very good yield. Similar reaction of $\mathbf{2}$ with pentafluorobenzoyl chloride provided the bis-amide 5c. Similarly, the reaction of $\mathbf{4}$ with corresponding fluorinated acid chlorides provided the tris-amide derivatives $\mathbf{6 a}-\mathbf{c}$ in good yield. 
Scheme 2. Synthesis of amide-amine building blocks from $N$-Boc- $\beta$-alanine and $N$-Boc-L-aspartic acid.

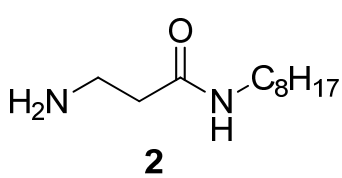

2<smiles>NC(CC(=O)Nc1ccccc1)C(=O)Nc1ccccc1</smiles>

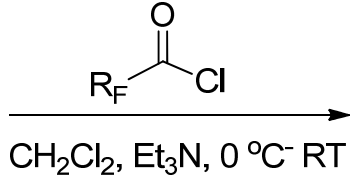<smiles>[R]ON(CC)OCC</smiles><smiles>[R]CNC(=O)CCNC(=O)O</smiles>

5a: $\mathrm{R}_{\mathrm{F}}=\mathrm{C}_{6} \mathrm{~F}_{13} \mathrm{CH}_{2} \mathrm{CH}_{2}-86 \%$

5b: $\mathrm{R}_{\mathrm{F}}=\mathrm{C}_{4} \mathrm{~F}_{9} \mathrm{CH}_{2} \mathrm{CH}_{2}-86 \%$

5c: $\mathrm{R}_{\mathrm{F}}=\mathrm{C}_{6} \mathrm{~F}_{5}-82 \%$<smiles>[R]C(=O)N[C@@H](CC(N)=O)C(=O)Nc1ccccc1</smiles>

6a: $\mathrm{R}_{\mathrm{F}}=\mathrm{C}_{6} \mathrm{~F}_{13} \mathrm{CH}_{2} \mathrm{CH}_{2^{-}} 77 \%$

6b: $\mathrm{R}_{\mathrm{F}}=\mathrm{C}_{4} \mathrm{~F}_{9} \mathrm{CH}_{2} \mathrm{CH}_{2}-61 \%$

6c: $\mathrm{R}_{\mathrm{F}}=\mathrm{C}_{6} \mathrm{~F}_{5^{-}} \quad 66 \%$

\subsubsection{Partially Fluorinated Urea-Amides}

The amide-amine building blocks (2 and 4) were also used for the preparation of partially fluorinated urea-amide derivatives, as described in Scheme 3. Compound 2, upon reacting with $1 \mathrm{H}, 1 \mathrm{H}, 2 \mathrm{H}, 2 \mathrm{H}$-perfluorooctyl isocyanate or $1 \mathrm{H}, 1 \mathrm{H}, 2 \mathrm{H}, 2 \mathrm{H}$-perfluorohexyl isocyanate, provided the urea-amide derivatives $\mathbf{7 a}$ or $\mathbf{7 b}$, respectively, in good yield. Similar reaction of $\mathbf{2}$ with pentafluorophenyl isocynante provided the bis-amide $\mathbf{7 c}$ in very good yield. Similarly, the reaction of compound $\mathbf{4}$ with corresponding fluorinated isocyanates provided the urea-bis-amide derivatives 8a-c in very good yield.

Scheme 3. Synthesis of amide-amine building blocks from $N$-Boc- $\beta$-alanine and $N$-Boc-L-aspartic acid.

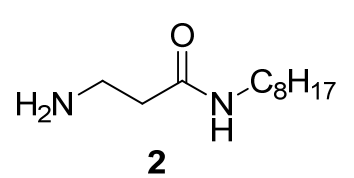<smiles>[R]N=C=O</smiles>

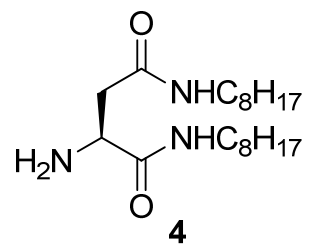

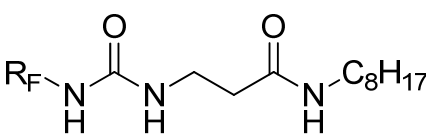

7a: $\mathrm{R}_{\mathrm{F}}=\mathrm{C}_{6} \mathrm{~F}_{13} \mathrm{CH}_{2} \mathrm{CH}_{2^{-}} 74 \%$

7b: $\mathrm{R}_{\mathrm{F}}=\mathrm{C}_{4} \mathrm{~F}_{9} \mathrm{CH}_{2} \mathrm{CH}_{2}-67 \%$

7c: $\mathrm{R}_{\mathrm{F}}=\mathrm{C}_{6} \mathrm{~F}_{5}-84 \%$

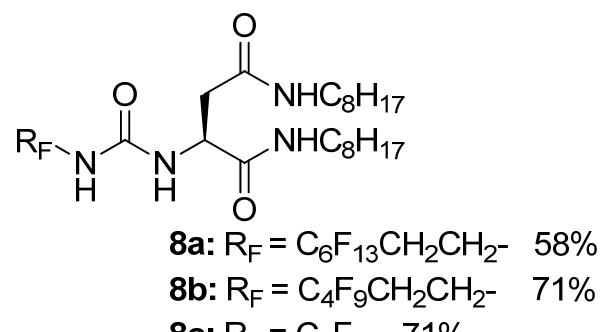

\subsection{Gelation in Organic Solvents}

The majority of these newly synthesized amide and urea-amide derivatives showed gelation properties in organic solvents during their work-up or isolation. A systematic study was then 
performed to examine the gelation behavior of these partially fluorinated amide or urea-amide derivatives in various organic solvents. A methodology was developed where gelation was attempted with varying amounts of organogelator with a matching a solvent system. A suitable solvent system selection is based on initial solubility experiments. In general, a gelator that is too soluble in an organic solvent will stay dissolved without forming a gel, even at high concentrations. If the gelator is not soluble in an organic solvent, and partially or fully dissolves at high temperature, it may precipitate again as the temperature is lowered. Ideally, the organogelators should dissolve in a solvent at a temperature close to its boiling point and assemble into a network upon cooling, leading to a single gel phase that does not move upon shaking or inverting the vial.

In a typical screening experiment, $0.5-4 \mathrm{mg} / \mathrm{mL}$ slurries of the organogelators in various organic solvents were prepared and heated with stirring at a temperature $5{ }^{\circ} \mathrm{C}$ below the boiling point of the solvent to induce full dissolution. Gelation occurred upon cooling, as was evident by significant increase in viscosity and/or the formation of a translucent-to-opaque appearance, without the formation of precipitate. The majority of the partially fluorinated amide and urea-amide derivatives showed gelation properties in a variety of organic solvents at remarkably low concentrations, ranging from 0.5 to $4 \mathrm{mg} / \mathrm{mL}$. The partially fluorinated amide derivative 5a showed gelation properties in acetone (2-3 $\mathrm{mg} / \mathrm{mL}$ ) and $n$-butanol $(3-4 \mathrm{mg} / \mathrm{mL}$ ) leading to partial gels. The corresponding perfluorobutyl derivative (5b) showed gelation in acetone at $3-4 \mathrm{mg} / \mathrm{mL}$ and was a clear solution in $n$-butanol at various concentrations. Both $\mathbf{5 a}$ and $\mathbf{5 b}$ existed either as clear solutions or slurries in THF, dichloromethane, methanol and toluene at various concentrations $(0.5-4 \mathrm{mg} / \mathrm{mL})$, and did not show any signs of gelation. Compound $\mathbf{5 c}$ did not gel under any conditions tested, indicating that a long-chain perfluoroalkyl group is probably needed to induce gelation of these bis-amide systems. We then examined the tris-amide systems $\mathbf{6 a - c}$ for their gelation abilities at various concentrations in a variety of organic solvents. Compound $6 \mathbf{a}$ gelled in acetone $(2 \mathrm{mg} / \mathrm{mL})$ and in $n$-butanol $(4 \mathrm{mg} / \mathrm{mL})$ to form opaque gels which were stable for several weeks at room temperature. Similarly, compound $\mathbf{6 b}$ formed stable hazy gels in acetone at $(3 \mathrm{mg} / \mathrm{mL})$ and in dichloromethane $(2 \mathrm{mg} / \mathrm{mL})$. Compound $\mathbf{6 c}$ formed gels in acetone at $(1-2 \mathrm{mg} / \mathrm{mL})$ and in methanol $(2 \mathrm{mg} / \mathrm{mL})$, indicating gelation could be induced on this tris-amide system even though it comprises a pentafluorophenyl group.

Gelation of urea-amide derivatives (7a-c and $\mathbf{8 a}-\mathbf{c}$ ) was then attempted in a variety of organic solvents under varying concentrations. Compound $7 \mathbf{a}$ gelled in THF $(2 \mathrm{mg} / \mathrm{mL})$ and acetone $(2 \mathrm{mg} / \mathrm{mL})$ forming stable gels, and partially gelled in $n$-butanol $(3 \mathrm{mg} / \mathrm{mL})$. Compound $7 \mathbf{b}$ gelled in acetone and THF ( $3 \mathrm{mg} / \mathrm{mL}$ ), leading to stable gels. The pentafluorophenyl urea-amide derivative $7 \mathbf{c}$ remained as slurry or solution in many organic solvents, although partial gel formation was observed in dichloromethane $(2 \mathrm{mg} / \mathrm{mL})$. The urea-bis-amide derivatives 8a-c showed excellent gelation properties in a variety of organic solvents. Compound 8a gelled in both acetone and dichloromethane at $2-3 \mathrm{mg} / \mathrm{mL}$ concentrations. Compound 8a also showed partial gelation in THF and $n$-butanol at 2-4 $\mathrm{mg} / \mathrm{mL}$ concentrations. Compound $\mathbf{8 b}$ gelled in dichloromethane $(2 \mathrm{mg} / \mathrm{mL})$ and acetone $(3 \mathrm{mg} / \mathrm{mL}$ ), leading to stable gels. Compound $\mathbf{8 c}$ showed excellent gelation behavior with a variety of organic solvents (toluene, THF, acetone, dichloromethane, $n$-butanol and methanol) at concentrations ranging from $0.5-3 \mathrm{mg} / \mathrm{mL}$, and formed stable gels in several instances. Table 1 summarizes some of the best conditions for gel formation, and the minimum concentration of gelator where stable gels were obtained. 
Table 1. Gelation properties of partially fluorinated amides $(\mathbf{5 a}-\mathbf{c}, \mathbf{6 a}-\mathbf{c})$ and urea-amides $(7 \mathbf{a}-\mathbf{c}, 8 \mathbf{a}-\mathbf{c})$.

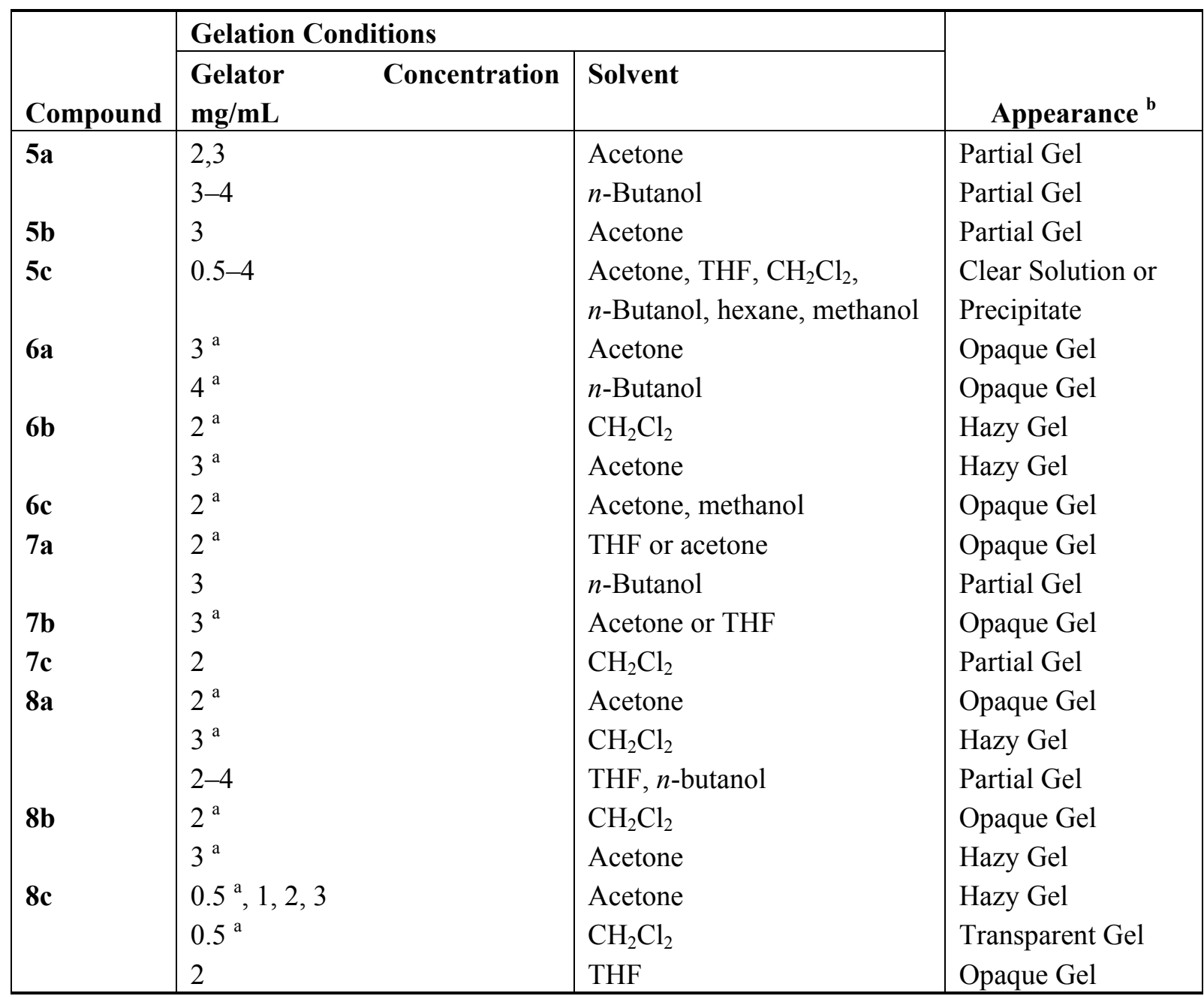

${ }^{\mathrm{a}}$ Minimum gel concentration for stable gel formation; ${ }^{\mathrm{b}}$ Hazy $=$ partially transparent, solid gel. Transparent $=$ completely transparent, solid gel. Opaque $=$ solid gel, not transparent. Partial gel = solid gel with some movement. Precipitate $=$ phase- separated liquid, with solid particles.

In general, we conclude that the partially fluorinated urea-amide derivatives $(7 \mathbf{a}-\mathbf{c}$ and $\mathbf{8 a}-\mathbf{c})$ are better gelators than the corresponding amide systems $(\mathbf{5 a}-\mathbf{c}$ and $\mathbf{6 a}-\mathbf{c})$, possibly due to the ability of the urea moieties to form directional hydrogen bonds. In order to further understand the influence of amide groups in the gelation ability of these urea-amide systems, we synthesized the corresponding urea-ester derivatives, which in general did not show any gelation properties under the same gelation conditions as the urea-amide systems. For example, urea-ester derivatives $\mathbf{9}$ and $\mathbf{1 0}$ (comparable to urea-amides $7 \mathbf{a}$ and $\mathbf{8 b}$ ) were synthesized via the esterification of $N$-Boc- $\beta$-alanine and $N$-Boc-L-aspartic acid with $n$-octanol, followed by deprotection and reaction of the amine with $1 \mathrm{H}, 1 \mathrm{H}, 2 \mathrm{H}, 2 \mathrm{H}$-perfluorooctyl isocyanate (Figure 3). Both $\mathbf{9}$ and $\mathbf{1 0}$ were oils and failed to gel in common organic solvents, indicating amide functionalities are desired in addition to the urea group in the backbone of these partially fluorinated compounds to obtain good gelation properties. 
Figure 3. Urea-ester derivatives from $N$-Boc- $\beta$-alanine and $N$-Boc-L-aspartic acid.<smiles>CCOC(=O)CCNC(=O)NC(CC(=O)OCC)C(=O)NCCC(F)(F)F</smiles>

\subsection{Surface Modification of Nonwoven Supports in Organic Solvents}

The concept of fabricating low surface energy surfaces via surface modification of nonwoven substrates using self-assembly of partially fluorinated urea or amide gelators has been described in our earlier reports [29,31]. The gelators self-assemble into fiber-like morphologies in solution, which grow sufficiently long and become entangled with one another, forming a gel with their solvent medium. In the presence of a solid or fibrous substrate, the gelation occurs on the surface of the substrate, creating a composite with an altered surface topology. Removal of the solvent from the gel composite leaves behind a network of assembled gelator fibers on the substrate, thus producing a composite surface with high surface area and roughness, which are desirable for superior hydrophobic/oleophobic properties.

A few of the partially fluorinated amide and urea-amide gelators that showed good gelation properties were gel-impregnated on nonwoven supports (Tyvek ${ }^{\circledR}$ polyethylene nonwoven fabric and Kolon ${ }^{\circledR}$ polyester fabric) in a suitable organic solvent. The nonwoven supports were then dried in a vacuum oven at room temperature to produce a composite of xerogel coated on the substrate. These surface-modified composites were then evaluated for hydrophobic and oleophobic properties. The tris-amide gelators (6a and 6b) were impregnated on the Tyvek ${ }^{\circledR}$ and Kolon ${ }^{\circledR}$ fabrics in acetone and dichloromethane, respectively, leading to the corresponding gel-impregnated composites. Similarly, the partially fluorinated urea-amide gelators (7a and 8c) that showed excellent gelation behavior were gel impregnated on both Tyvek ${ }^{\circledR}$ and Kolon ${ }^{\circledR}$ fabrics in acetone, leading to the corresponding surfacemodified composites. Examination of these composites by SEM showed a nanofibrous network of gelators impregnated on the fabric surface, vastly different from the micro structured surfaces of Tyvek $^{\circledR}$ and Kolon ${ }^{\circledR}$ fabrics [31]. Figure 4 shows SEMs at 2,000 $\times$ and 10,000 $\times$ magnification of the Kolon ${ }^{\circledR}$ fabric surface-treated with gelator $7 \mathbf{a}$ with an average fiber diameter of $142 \mathrm{~nm}$.

Figure 4. SEMs of composite prepared by impregnation of gelator 7a on Kolon ${ }^{\circledR}$ fabric at $2,000 \times($ left) and 10,000× (right) magnifications.
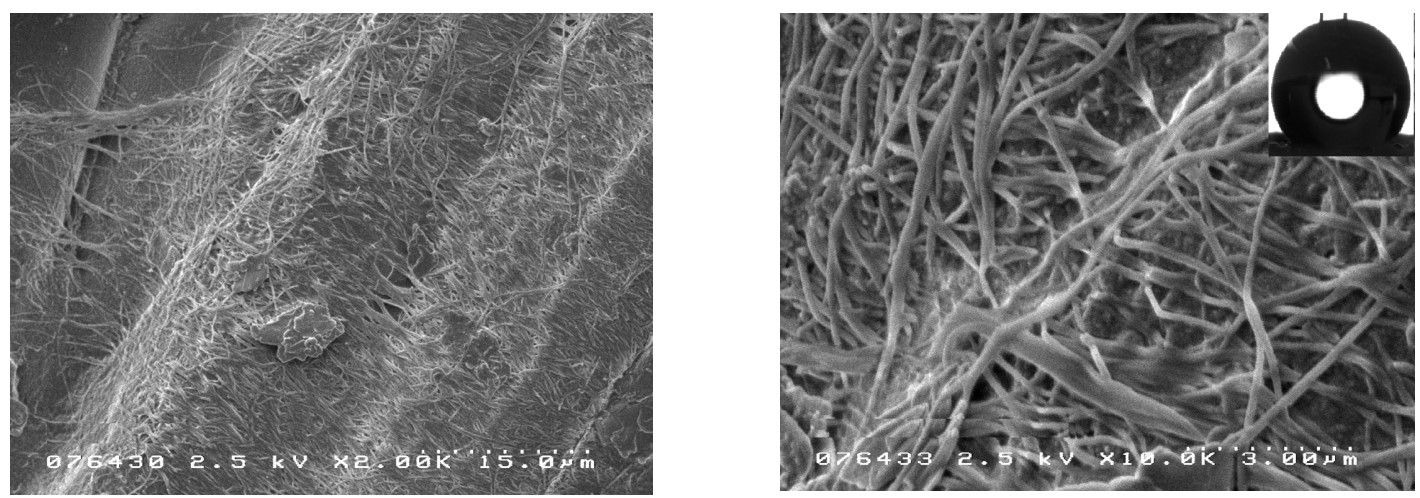
The composites produced by gel-impregnation of fluorinated organogelators were evaluated for water/oil repellency via dynamic contact angle measurement for water and hexadecane. Table 2 summarizes the results of contact angle measurements for the composites and those for untreated controls (Tyvek ${ }^{\circledR}$ and Kolon ${ }^{\circledR}$ fabrics). The results indicate that the composite materials produced by the gel impregnation of fluorinated gelators $6 \mathbf{a}, 6 \mathbf{b}, 7 \mathbf{a}, 8 \mathbf{a}$ and $8 \mathbf{c}$ exhibit significantly higher water advancing contact angles than the untreated controls. The higher water contact angles observed for these surfaces suggest increased surface roughness in addition to the incorporation of hydrophobic alkyl or fluoroalkyl functionalities. The inset in Figure 4 shows a water droplet sitting on the Kolon ${ }^{\circledR}$ fabric surface treated with gelator $7 \mathbf{a}$, with an advancing contact angle of $140^{\circ}$. The effect of surface structure created by gel-impregnation is particularly important as clear solutions (no gel with solvents chosen) of urea-amide gelators dip-coated on nonwoven substrates showed relatively inferior performance (water advancing contact angle $<117^{\circ}$ ). The treated composites derived from gelators $6 \mathbf{6}$, 6b and 7a (comprising perfluoroalkyl chains) also showed a high advancing contact angle for hexadecane, indicating excellent oil repellency. Surprisingly, the pentafluorophenyl-derived urea-amide derivative 8c, which showed excellent gelation characteristics, also showed excellent water advancing and receding contact angles (superhydrophobic), but extremely poor oil repellency, as hexadecane penetrated into the substrate within a few seconds. This observation is consistent with the need for long-chain perfluoroalkyl groups with terminal $\mathrm{CF}_{3}$ groups in the gelator molecule in order to provide oil repellency [35]. Surface structure created by the gelator and pentafluorophenyl groups or hydrocarbon alkyl groups in the gelator backbone are not sufficient for imparting oil repellency to the treated composites.

Table 2. Water and hexadecane contact angles of treated nonwoven composites prepared via gelation in organic solvents

\begin{tabular}{|c|c|c|c|c|c|}
\hline \multirow[b]{3}{*}{ Composite } & \multirow{3}{*}{$\begin{array}{l}\text { Average Amount of } \\
\text { Dry Gel Impregnated } \\
\text { per Unit Area, g/cm² }\end{array}$} & \multicolumn{4}{|c|}{ Contact angle $^{\dagger}$} \\
\hline & & \multicolumn{2}{|c|}{ Water } & \multicolumn{2}{|c|}{ Hexadecane } \\
\hline & & Adv CA & $\operatorname{Rec} C A$ & Adv CA & $\operatorname{Rec} C A$ \\
\hline 6a on Tyvek ${ }^{\circledR}$ & 0.0014 & $141 \pm 4$ & $134 \pm 1$ & $89 \pm 2$ & $64 \pm 1$ \\
\hline 6a on Kolon ${ }^{\circledR}$ & 0.0027 & $157 \pm 6$ & $144 \pm 3$ & $94 \pm 5$ & $61 \pm 3$ \\
\hline 6b on Tyvek ${ }^{\circledR}$ & 0.0041 & $128 \pm 4$ & $112 \pm 3$ & $60 \pm 2$ & $30 \pm 3$ \\
\hline 6b on Kolon ${ }^{\circledR}$ & 0.0012 & $134 \pm 1$ & $118 \pm 2$ & $59 \pm 4$ & $29 \pm 5$ \\
\hline 7a on Tyvek ${ }^{\circledR}$ & 0.0015 & $140 \pm 4$ & $126 \pm 1$ & $84 \pm 4$ & $43 \pm 1$ \\
\hline 7a on Kolon ${ }^{\circledR}$ & 0.0030 & $154 \pm 3$ & $138 \pm 2$ & $87 \pm 4$ & $42 \pm 2$ \\
\hline 8c on Tyvek ${ }^{\circledR}$ & 0.0013 & $161 \pm 1$ & $148 \pm 1$ & \multicolumn{2}{|c|}{ Absorbed in $25 \mathrm{~s}$} \\
\hline $8 \mathbf{c}$ on $\operatorname{Kolon}^{\circledR}$ & 0.0037 & $158 \pm 2$ & $145 \pm 1$ & \multicolumn{2}{|c|}{ Absorbed in $30 \mathrm{~s}$} \\
\hline Untreated Tyvek $^{\circledR}$ & --- & $108 \pm 1$ & $78 \pm 1$ & \multicolumn{2}{|c|}{ Absorbed on contact } \\
\hline Untreated Kolon $^{\circledR}$ & $-\cdots$ & $115 \pm 4$ & $85 \pm 4$ & \multicolumn{2}{|c|}{ Absorbed on contact } \\
\hline
\end{tabular}

${ }^{\dagger}$ Average of 3 runs at different positions on each sample.

\section{Conclusions}

A new class of partially fluorinated amides and urea-amides useful as organogelators was synthesized from amino acid building blocks. These derivatives showed good gelation properties in a 
variety of organic solvents, and in general the urea-amide derivatives were better gelators than the corresponding bis- or tris-amide derivatives. Analogous urea-ester derivatives failed to gel in any organic solvents, indicating a combination of urea and amide functionalities, or multiple amide groups, are desirable to induce gelation. Good organogelators derived from the initial screening were evaluated for surface modification of nonwoven substrates, and surface analysis indicated a gel-impregnated surface with porous microstructure morphology. Composites obtained via impregnation by gelators provided surfaces with excellent hydrophobic properties. The nonwoven surfaces impregnated with perfluoroalkyl-containing organogelators showed excellent oleophobic behavior. Superior hydrophobic and oleophobic behavior exhibited by these composites were attributed to a combination of micro-structured surface morphology created by the xerogel and the presence of fluorocarbon/hydrocarbon functionalities in the gelator backbone.

\section{Acknowledgments}

We gratefully acknowledge Stefan Reinartz of DuPont Performance Coatings (DPC) for helpful insight into this research and Liang Liang of DuPont Corporate Center for Analytical Sciences (CCAS) for microscopy.

\section{References}

1. Terech, P.; Weiss, R.G. Low-molecular mass gelators of organic liquids and the properties of their gels. Chem. Rev. 1997, 97, 3133-3160.

2. Weiss, R.G., Terech, P., Eds.Molecular Gels: Materials with Self-Assembled Fibrillar Networks; Springer: Dordrecht, The Netherlands, 2006.

3. George M.; Weiss, R.G. Molecular organogels. Soft matter comprised of low-molecular-mass organic gelators and organic liquids. Acc. Chem. Res. 2006, 39, 489-497.

4. van Esch, J.H.; Feringa, B.L. New functional materials based on self-assembling organogels: From serendipity towards design. Angew. Chem. Int. Ed. 2000, 39, 2263-2266.

5. Srinivasan, S.; Babu, P.A.; Mahesh, S.; Ajayaghosh, A. Reversible self-assembly of entrapped fluorescent gelators in polymerized styrene gel matrix: Erasable thermal imaging via recreation of supramolecular architectures. J. Am. Chem. Soc. 2009, 131, 15122-15123.

6. Sahoo, S.; Kumar, N.; Bhattacharya, C.; Sagiri, S.S.; Jain, K.; Pal, K.; Ray, S.S.; Nayak, B. Organogels: Properties and applications in drug delivery. Des. Monomers Polym. 2011, 14, 95-108.

7. Debnath, S.; Shome, A.; Dutta, S.; Das, Prasanta, K. Dipeptide-based low-molecular-weight efficient organogelators and their application in water purification. Chem.-A Eur. J. 2008, 14, 6870-6881.

8. Smith, D.K. Molecular gels: Nanostructured soft materials. In Organic Nanostructures; Atwood, J.L., Steed, J.W., Eds.; Wiley-VCH: Weinheim, Germany, 2008; pp. 111-154.

9. Hughes, N.E.; Marangoni, A.G.; Wright, A.J.; Rogers, M.A.; Rush, J.W.E. Potential food applications of edible oil organogels. Trends Food Sci. Technol. 2009, 20, 470-480.

10. Fages, F.; Voegtle, F.; Zinic, M. Systematic design of amide- and urea-type gelators with tailored properties. Top. Curr. Chem. 2005, 256, 77-131. 
11. Allix, F.; Curcio, P.; Pham, Q.; Pickaert, G.; Jamart-Grégoire, G. Evidence of intercolumnar $\pi-\pi$ stacking interactions in amino-acid-based low-molecular-weight organogels. Langmuir 2010, 26, $16818-16827$.

12. Song, B.; Wei, H.; Wang, Z.; Zhang, X.; Smet, M.; Dehae, W. Supramolecular nanofibers by self-organization of bola-amphiphiles through a combination of hydrogen bonding and $\pi-\pi$ stacking interactions. Adv. Mater. 2007, 19, 416-420.

13. Suzuki, M.; Hanabusa, K. Polymer organogelators that make supramolecular organogels through physical cross-linking and self-assembly. Chem. Soc. Rev. 2010, 39, 455-463.

14. de Loos, M.; Feringa, B.L.; van Esch, J.H. Design and application of self-assembled low molecular weight hydrogels. Eur. J. Org. Chem. 2005, 3615-3631.

15. Hirst, A.R.; Coates, I.A.; Boucheteau, T.R.; Miravet, J.F.; Escuder, B.; Castelletto, V.; Hamley, I.W.; Smith, D.K. Low-molecular-weight gelators: Elucidating the principles of gelation based on gelator solubility and a cooperative self-assembly model. J. Am. Chem. Soc. 2008, 130, 9113-9121.

16. Liu, J.; Ma, J.; Chen, C. Structure-property relationship of a class of efficient organogelators and their multistimuli responsiveness. Tetrahedron 2011, 67, 85-91.

17. Mohmeyer, N.; Schmidt, H. Synthesis and structure-property relationships of amphiphilic organogelators. Chem. Eur. J. 2007, 13, 4499-4509.

18. Raynal, M.; Laurent, B. Organogel formation rationalized by Hansen solubility parameters. Chem. Commun. 2011, 47, 8271-8273.

19. Srinivasan, S.; Praveen, V.K.; Philip, R.; Ajayaghosh, A. Bioinspired superhydrophobic coatings of carbon nanotubes and linear $\pi$ systems based on the "bottom-up" self-assembly approach. Angew. Chem. Int. Ed. 2008, 47, 5750-5754.

20. Ren, C.L.; Xu, S.Y.; Xu, J.; Chen, H.Y.; Zeng, H.Q. Planar macrocyclic fluoropentamers as supramolecular organogelators. Org. Lett. 2011, 13, 3840-3843.

21. Hanabusa, K. Development of organogelators based on supramolecular chemistry. In Macromolecular Nanostructured Materials; Ueyama, N., Harada, A., Eds.; Springer Series in Materials Science: Dordrecht, The Netherlands, 2004; Volume 78, pp. 118-137.

22. Shi, C.; Huang, Z.; Kilic, S.; Xu, J.; Enick, R.M.; Beckman, E.J. Carr, A.J.; Melendez, R.E.; Hamilton, A.D. The gelation of $\mathrm{CO}_{2}$ : A sustainable route to the creation of microcellular materials. Science 1999, 286, 1540-1543.

23. Zhou, Y.; Yi; T.; Li, T.; Zhou, Z.; Li, F.; Huang, W.; Huyang, C. Morphology and wettability tunable two-dimensional superstructure assembled by hydrogen bonds and hydrophobic interactions. Chem. Mater. 2006, 18, 2974.

24. Yamanaka, M.; Sada, K.; Miyata, M.; Hanabusa, K.; Nakano, K. Construction of superhydrophobic surfaces by fibrous aggregation of perfluoroalkyl chain-containing organogelators. Chem. Commun. 2006, 21, 2248-2250.

25. Nakajima, A.; Hashimoto, K.; Watanabe, T. Some Recent studies on super-hydrophobic films. Monateshefte Fur Chemie 2001, 132, 31-41.

26. Feng, L.; Li, S.; Li, Y.; Li, H.; Zhang, L.; Zhai, J.; Yanlin, S.; Liu, B.; Jiang, L.; Zhu, D. Super-hydrophobic surfaces: from natural to artificial. Adv. Mater. 2002, 14, 1857-1864. 
27. Chen, W.; Fadeev, A.Y.; Che Hsieh, M.; Oner, D.; Youngblood, J.; McCarthy, T. Ultrahydrophobic and ultralyophobic surfaces: Some comments and examples. Langmuir 1999, 15, 3395-3399.

28. Barthlott, W.; Neinhuis, C. Purity of the sacred lotus, or escape from contamination in biological surfaces. Planta 1997, 202, 1-8.

29. Nun, E.; Oles, M.; Schleich, B. Lotus-effect-surfaces. Macromol. Symp. 2002, 187, 677-682.

30. Gould, P. Smart clean surfaces. Mater. Today 2003, 6, 44-48.

31. Raghavanpillai, A.; Reinartz, S.; Hutchenson, K.W. Hydrophobic and oleophobic surface modification using fluorinated bis-urea and bis-amide gelators. J. Fluor. Chem. 2009, 130, 410-417.

32. Schoonbeek, F.S.; Van Esch, J.H.; Hulst, R.; Kellogg, R.M.; Feringa, B.L. Geminal Bis-ureas as gelators for organic solvents: Gelation properties and structural studies in solution and in the gel state. Chem. Eur. J. 2000, 6, 2633-2643.

33. Raghavanpillai, A.; Franco, V.A.; Meredith, W.E. Hydrophobic and oleophobic surface modification using gelling agents derived from amino acids. J. Fluor. Chem. 2012, 135, 187-194.

34. Jouani, A.M.; Szonyi, F.; Cambon, A. Synthesis of 2-F-alkylethyl isocyanates. J. Fluor. Chem. 1992, 56, 85-92.

35. Honda, K.; Morita, M.; Otsuka, H.; Takahara, A. Molecular aggregation structure and surface properties of poly(fluoroalkyl acrylate) thin films. Macromolecules 2005, 38, 5699-5705.

(C) 2012 by the authors; licensee MDPI, Basel,Switzerland. This article is an open access article distributed under the terms and conditions of the Creative Commons Attribution license (http://creativecommons.org/licenses/by/3.0/). 